\title{
RECURSOS GENÉTICOS Y PUEBLOS INDÍGENAS: LA TESIS DE LA PROPIEDAD CULTURAL INDÍGENA FRENTE AL DOMINIO PÚBLICO
}

\author{
Salvador Millaleo Hernández ${ }^{1}$ \\ Resumen: El artículo analiza la posición de los pueblos indígenas y sus fundamentos ético-políticos respecto de la protección \\ debida a los recursos genéticos vinculados a los conocimientos tradicionales. Dicha posición ha reivindicado la propiedad \\ cultural de índole colectiva respecto de dichos recursos, diferenciándose explícitamente de la solución que entiende que estos \\ deberían caer en el dominio público. Para ello, se esboza una concepción de propiedad que tiene otras determinaciones respecto \\ de la propiedad occidental. La postura de los pueblos indígenas ha sido sostenida de manera amplia y consistente durante \\ décadas, y ha llegado a plasmarse en el régimen internacional de derechos humanos a través de la Declaración de Derechos \\ de los Pueblos Indígenas de la ONU; pero, en las negociaciones en la OMPI, no ha logrado abrirse paso frente a sus críticos \\ y a los intereses de las empresas y los Estados.
}

Palabras clave: recursos genéticos, conocimientos tradicionales, propiedad cultural, pueblos indígenas, dominio público

Genetic resources and indigenous peoples: the thesis of indigenous cultural property in the face of public domain

\begin{abstract}
The article analyzes the position of indigenous peoples and their ethical-political foundations regarding due protection of genetic resources linked to traditional knowledge. This position has claimed cultural property of a collective nature with respect to these resources, differing explicitly from the solution that understands that these should fall into the public domain. For this purpose, a conception of property with other determination factors, compared to Western property, is outlined. The position of indigenous peoples has been widely and consistently sustained for decades and has come to be included in the international human rights regime, through the UN Declaration on the Rights of Indigenous Peoples; but, in the negotiations at WIPO, it has not managed to break past its critics and the interests of companies and states.
\end{abstract}

Keywords: genetic resources, traditional knowledge, cultural property, indigenous peoples, public domain

\section{Recursos genéticos e povos indígenas: a tese da propriedade cultural indígena frente ao domínio público}

Resumo: $\mathrm{O}$ artigo analisa a posição dos povos indígenas e seus fundamentos ético-políticos a respeito da proteção devida aos recursos genéticos vinculados aos conhecimentos tradicionais. Dita posiçáo reivindicou a propriedade cultural de índole coletiva a respeito de tais recursos, diferenciando-se explicitamente da soluçáo que entende que deveriam cair em domínio público. Para isso, se esboça uma concepção de propriedade que tem outras determinaçóes a respeito da propriedade ocidental. A postura dos povos indígenas tem sido mantida de maneira ampla e consistente durante décadas e chegou a se refletir no regime internacional de direitos humanos através da Declaração de Direitos dos Povos Indígenas da ONU; porém nas negociaçôes na OMPI não conseguiu se impor diante de seus críticos e aos interesses das empresas e dos Estados.

Palavras-chave: recursos genéticos, conhecimentos tradicionais, propriedade cultural, povos indígenas, domínio público

\footnotetext{
${ }^{1}$ Centro de Derechos Humanos, Facultad de Derecho, Universidad de Chile, Chile Correspondencia: smillaleo@hotmail.com
} 


\section{Introducción}

El objeto de este artículo es analizar las posturas de los pueblos indígenas sobre la regulación del acceso a los recursos genéticos, en las que han defendido la tesis de la propiedad cultural sobre los recursos vinculados a sus conocimientos tradicionales y la han reclamado como derecho fundamental en diversos foros internacionales.

Este fue uno de los puntos más álgidos de los debates del Comité Intergubernamental sobre Propiedad Intelectual y Recursos Genéticos, Conocimientos Tradicionales y Folclore (IGC) de la Organización Mundial de la Propiedad Intelectual (OMPI), y forma parte del desacuerdo sobre los contenidos a nivel internacional y en los debates internos para proteger los intereses indígenas en los recursos genéticos.

La búsqueda de soluciones de protección frente a los derechos soberanos y de propiedad privada sobre aquellos recursos, incluso cuando se han impulsado regímenes sui generis de propiedad intelectual indígena, olvidan los fundamentos ético-políticos de las demandas indígenas que asientan sus preferencias y posiciones en los debates domésticos e internacionales.

\section{Metodología}

A partir de la revisión de las declaraciones de grupos indígenas y las explicaciones teóricas disponibles, reconstruiremos analíticamente los fundamentos ético-políticos de la propiedad cultural indígena, como posición contraria a la solución de la pertenencia al dominio público de los recursos genéticos, para culminar en una breve revisión de las críticas a dicha posición.

1. El estatus de los recursos genéticos entre bienes comunes y privados

Uno de los debates más relevantes de las últimas décadas del ámbito de la propiedad intelectual y de las relaciones del derecho con las ciencias de la vida, ha sido el estatus de los recursos genéticos, en cuanto a la posibilidad de acceder legítimamente a ellos y los derechos de control sobre ellos. Dichos recursos han sido los protagonistas de la investigación y revolución de las biotecno- logías de los últimos tiempos. Dicha revolución y la de saberes relacionados, como la genética, genómica, bioinformática y biología sintética, han hecho que los recursos genéticos se vuelvan cada vez más valiosos, debido a su importancia para el desarrollo agrícola, la seguridad alimentaria, salud pública, cambio climático y sustentabilidad ambiental(1).

Una cuestión debatida son las formas de protección que se deben acordar internacionalmente e implementar cuando están en juego los intereses de los pueblos indígenas(2).

Los recursos genéticos han pasado por diversos cambios de estatus en cuanto a su acceso y control, provocando que los pueblos indígenas incorporen un discurso sobre los derechos que reivindican sobre ellos.

\section{A. Recurso común:}

Los recursos genéticos se han recopilado y conservado durante la mayor parte de la historia del derecho como recursos comunes. Esto significó un libre acceso a dichos recursos, de manera que cualquier persona podía acceder a ellos y no eran propiedad de nadie(3:319).

En dicho régimen, aunque un espécimen particular de planta o animal podían ser apropiables, los recursos genéticos se consideran como bienes comunes. Este régimen determinó el acceso abierto, lo que significaba que los Estados generalmente no restringían a otros la obtención de muestras de recursos genéticos(4). Antes que estar formalmente reconocido, dicho régimen se plasmaba en una práctica general uniforme y consistente de acceso y uso de los recursos vegetales(5).

El acceso libre será controvertido ya que, se sostiene, favorece a los países ricos e industrializados, por tener mayores conocimientos y los medios financieros y tecnológicos para acceder y explotar esos recursos(6).

\section{B. Derechos estatales soberanos:}

El enfoque anterior dará paso a una regulación que excluye o controla el libre acceso desde los países de origen mediante los derechos soberanos estatales a fines del siglo XX. Este segundo enfoque 
será esbozado en la Resolución № 3016(XXVII) sobre "Soberanía Permanente sobre los Recursos Naturales de los Países en Desarrollo", de 1972, y tendrá su punto culminante en el Convenio sobre la Diversidad Biológica de 1992 — así como en el Protocolo de Nagoya-, en el que se determinará que la autoridad para determinar el acceso a los recursos genéticos recae en los gobiernos nacionales y está sujeta a la legislación nacional. Los acuerdos internacionales que adoptan un enfoque de derechos estatales soberanos establecen que el acceso a los recursos genéticos está sujeto al consentimiento previo del Estado proveedor, que puede negociar las condiciones para compartir los beneficios que surjan de su posterior explotación. Este control es una proyección de la soberanía, en cuanto los recursos naturales que caen dentro de los límites de un Estado están sujetos al alcance de su jurisdicción. Es el Estado el que debe decidir qué régimen legal establecerá para el acceso o uso de los recursos genéticos, incluyendo la posibilidad de derechos de propiedad $(7: 4)$.

\section{Derechos propietarios privados:}

La revolución de las biotecnologías ha producido una presión para la constitución de derechos de propiedad privada sobre los recursos genéticos. Los actores de las innovaciones biotecnológicas exigieron derechos de propiedad en respuesta a la posibilidad de aumentar el valor de los recursos genéticos y el deseo de apropiarse de ese valor(4:287). Con la explosión del desarrollo de las biotecnologías se han planteado reclamos garantizados de propiedad privada (salvo el genoma humano), en concordancia con el acuerdo sobre Trade Related Aspects of Intellectual Property (TRIPS), a través de dos formas principales del derecho de propiedad intelectual, a saber, las patentes y los derechos de obtención de variedades vegetales.

La combinación de los sistemas basados en patentes de los países desarrollados y los sistemas basados en soberanía estatal de los países en desarrollo, al permitir o afirmar los derechos de propiedad sobre el material genético, han generado una privatización extensiva de los recursos genéticos(5:642).
2. La propiedad cultural de los pueblos indígenas sobre sus recursos genéticos

De acuerdo con la visión de Vandana Shiva, la biotecnología hace posible colonizar y controlar la naturaleza, la cual, en cambio, es considerada desde las cosmovisiones indígenas como autónoma, libre y auto regenerativa. El reduccionismo de la ciencia permite el acceso del capital a la última frontera de la vida(8:27). El avance de derechos propietarios sobre los recursos genéticos ha despertado demandas de restablecer el lugar de los recursos genéticos como recurso común de la humanidad, más allá de las jurisdicciones estatales. Sin embargo, uno de los sectores más afectados por aquel avance — los pueblos indígenas - han preferido demandar el reconocimiento de su propiedad cultural sobre los recursos genéticos.

Los sistemas de derechos de propiedad intelectual han sido cuestionados por los pueblos indígenas por promover la comercialización y la mercantilización de los conocimientos tradicionales, en términos no equitativos con sus creadores. Se critica que los indígenas, en general, no están suficientemente informados sobre los mecanismos legales de la propiedad intelectual, siendo muy costoso el ejercicio de sus derechos e intereses dentro de ellos. Sobre todo, se cuestiona que los sistemas de derechos de propiedad intelectual son incompatibles con las culturas indígenas, ya que se basan en la creatividad y apropiación individual(9).

Los pueblos indígenas han sostenido una estrategia doble para la protección de sus conocimientos tradicionales y manifestaciones culturales asociadas a los recursos genéticos, frente a los riesgos de protección ineficaz y favoreciendo su alienación por los derechos de propiedad intelectual de su apropiación. En primer lugar, una estrategia no propietarista, que ha empleado el concepto de "patrimonio cultural inmaterial", bajo el marco de las responsabilidades de los Estados en el contexto de las convenciones de la UNESCO, para salvaguardar dicho patrimonio, con el objeto de que se adopten medidas especiales de protección.

En segundo lugar, y esta ha sido la vía principal, los pueblos indígenas han usado una estrategia propietarista que busca se reconozcan derechos de control sobre los recursos genéticos, conoci- 
mientos tradicionales y expresiones culturales tradicionales(10). Dicha estrategia persigue, en el corto plazo, determinar que los conocimientos tradicionales indígenas no pertenecen al dominio público, mientras que, en el largo, que se reconozca que los conocimientos tradicionales son formas de propiedad intelectual de naturaleza colectiva $(11,12)$.

La propiedad indígena deriva de su derecho a la libre determinación, y se construye a partir de principios de lo colectivo o lo comunitario $(11: 7,13)$. La relación de los pueblos indígenas con los recursos naturales y sus conocimientos, incluyendo los recursos genéticos, es parte de una conexión espiritual, cultural y, en ocasiones, religiosa entre el pueblo y su territorio, de manera que privarlos de los recursos genéticos conectados a ellos constituye una violación de los derechos fundamentales de esos pueblos a su libre determinación(14:23). La propiedad cultural sobre su patrimonio cultural, conocimientos tradicionales y recursos genéticos vinculados a estos derivan directamente de los derechos de los pueblos indígenas a sus territorios(15:9).

La propiedad indígena se basa en la interconectividad de los objetos que forman parte de las culturas indígenas, de manera que los diversos elementos específicos están conectados a valores fundamentales para esas culturas. De allí que muchos objetos intangibles de los conocimientos estén vinculados a valores sagrados propios de las tradiciones indígenas (16:40-41).

El fundamento de la propiedad indígena establece como base el carácter de pueblo - o inclusive de nación- de los pueblos indígenas, de manera que dicho estatus, reconocido por el derecho internacional de los derechos humanos (Convenio 169 de la OIT, art. 1), hace que ciertos recursos y expresiones, tangibles e intangibles, están sujetos a la propiedad cultural de los pueblos indígenas, porque son inherentes a las identidades culturales de dichos pueblos e indispensables para su sobrevivencia cultural(17:1028).

Las nociones de "propiedad" en las sociedades indígenas varían considerablemente respecto de los conceptos occidentales. Con frecuencia no se acepta la propiedad en el sentido de un dere- cho que es disponible o enajenable. La noción de "propiedad cultural indígena" se define a partir de una concepción relacional de la propiedad, que se refiere al equilibrio en las relaciones de intereses de todos los involucrados antes que en la estabilización de las posibilidades de control y exclusión de unos por otros que implica la visión occidental de la propiedad, y en la que el dominio tiene un carácter fluido y dinámico, marcada por deberes sociales de índole fiduciaria, es decir, obligaciones derivadas de la propiedad concebida como forma de custodia de los objetos, en lugar de concebirse como poder de exclusión(17:1027-9). Los pueblos indígenas están imbuidos por nociones tales como la custodia, tutela y administración, antes que por la capacidad de enajenación o alienación de lo propio. Los pueblos indígenas a menudo se ven a sí mismos como guardianes y administradores de la naturaleza, con la cual sostienen relaciones de armonía y de equilibrio como componentes del Cosmos, de acuerdo con la mayoría de las cosmologías indígenas. Sin embargo, los pueblos indígenas no están satisfechos solo con el papel de custodios o administradores de la biodiversidad, si no se reconoce la naturaleza inalienable de su relación con su territorio y sus recursos, y su derecho a utilizar estos recursos(18).

El objeto de las demandas indígenas propietaristas consiste en los conocimientos tradicionales. La definición de conocimientos tradicionales siempre ha tenido como uno de sus ejemplos más distinguibles a los recursos genéticos a que están asociados. Esto se debe a que se ha sostenido que "los pueblos indígenas tienen conocimiento sobre las propiedades de los recursos genéticos, los ecosistemas de los que forman parte, sus medios de reproducción, las formas de cultivo y, lo que es más importante, sus usos potenciales."(10:318). Se trata de los casos en los cuales dichos recursos genéticos están de alguna manera relacionados con el conocimiento tradicional o, más en general, manifiestan las ciencias, las tecnologías y la cultura indígenas, han sido identificados, conservados, cultivados o utilizados de alguna manera por los pueblos indígenas(10:319).

Para los conocimientos tradicionales vinculados a los recursos genéticos, la propiedad implica la participación de los pueblos indígenas que los sostienen en los mecanismos para garantizar ac- 
ceso a estos, de manera que puedan beneficiarse de ellos y cuidar de su adecuado uso, en las condiciones que derivan de sus tradiciones, creencias y valores(17:1102). Por otro lado, en cuanto propiedad, la titularidad de los pueblos indígenas sobre sus conocimientos tradicionales puede significar excluir a otros de su uso, reproducción y diseminación(19:173), otorgando a los pueblos indígenas control para defender sus culturas y formas de vida(20). Las facultades y alcances de la propiedad cultural quedan sujetos a la regulación del derecho consuetudinario propio de los pueblos indígenas respectivos(21), no quedando, de esa manera, al simple arbitrio de las autoridades indígenas.

La determinación propietaria de los conocimientos tradicionales permite una defensa vigorosa contra diversas formas de apropiación no consentida, como el mal uso de los genes humanos indígenas o la apropiación de recursos genéticos animales o vegetales relacionados con los pueblos indígenas, a través de sus conocimientos tradicionales, así como para proteger directamente a dichos conocimientos.

En su específica aplicación a los recursos genéticos, David Bondía ha denominado a los reclamos indígenas de propiedad "derechos emergentes bioculturales", los cuales establecen el vínculo entre los pueblos indígenas y el respeto a sus ecosistemas(22).

Los recursos genéticos reflejan años de ingenio y habilidad que han resultado en su composición genética actual, a través de una cuidadosa dependencia y respuestas a patrones ecológicos y selecciones de mutaciones accidentales en la naturaleza, realizadas con base en prácticas tradicionales de mejoramiento por los pueblos indíge$\operatorname{nas}(23: 204)$.

El control de los pueblos indígenas también se justifica en sus conocimientos para manejar los ecosistemas locales, desarrollando a largo de muchos años conocimientos para sustentar la vida vegetal y animal.

En la Cumbre de la Tierra, en 1992, se firmó, por representantes de la Declaración de Kari-Oca, la Carta de la Tierra de los Pueblos Indígenas, y tiene una determinación clara sobre la propiedad intelectual indígena sobre los recursos genéticos:

"102. Como creadores y transmisores de civilización que han dado y continúan compartiendo su conocimiento, experiencia y valores con la humanidad, requerimos que nuestro derecho a la propiedad intelectual y cultural sea garantizado y que el mecanismo para cada aplicación esté en favor de nuestros pueblos y sea estudiado a fondo e implementado. Este respeto debe incluir el derecho sobre recursos genéticos, bancos genéticos, biotecnología y conocimiento de programas de biodiversidad"(24).

En 1993, la Declaración de Mataatua, realizada en Whakatane, Aotearoa/Nueva Zelanda, señala que: "Declaramos que los Pueblos Indígenas del Mundo tienen el derecho de autodeterminación y a ejercer sus derechos deben ser reconocidos como los dueños exclusivos de su propiedad cultural e intelectual" (25).

En la Declaración de Kimberley, en 2002, se afirmó que:

"Nuestro conocimiento no es del dominio público, es propiedad cultural e intelectual colectiva, protegido bajo nuestro derecho consuetudinario. El uso no autorizado y la apropiación indebida del conocimiento son una usurpación"(26).

El Foro Internacional Indígena sobre Biodiversidad, en la COP 8 de 2006, afirmó que los derechos indígenas "incluyen nuestros intereses de propiedad sobre nuestras tierras, aguas, territorios y recursos naturales, incluidos nuestros recursos genéticos"(27); ratificando de esa manera una postura consistente en todos los encuentros de las partes de la Convención sobre la Diversidad Biológica.

Más recientemente, en 2017, en la Declaración de la Alianza de los Guardianes e Hijos de la Madre Tierra de Brasilia, se hizo un llamado a:

"Crear mecanismos y legislaciones que garanticen la aplicación del Consentimiento Libre, Previo e Informado (CLPI) con el fin de proteger los derechos de propiedad intelectual indígena, en lo que respecta a las dimensiones espirituales, sagradas 
y secretas de sus conocimientos. Los Estados deben impedir la apropiación ilegal y ofensiva de los saberes tradicionales. Las disposiciones del Consentimiento Libre, Previo e Informado deben ser aplicadas dentro del contexto del acceso a este conocimiento y del compartir de los beneficios recibidos"(28).

En las negociaciones de las Naciones Unidas, en torno a la Declaración de Derechos de los Pueblos Indígenas, se agregó en 2004 la propuesta de reconocimiento de la propiedad intelectual indígena. Esto se relacionaba con las posturas de los representantes indígenas en las negociaciones del comité de trabajo intergubernamental de la OMPI/WIPO sobre recursos genéticos, conocimientos tradicionales y expresiones culturales tradicionales.

El texto final que aparece en la Declaración de Derechos de los Pueblos Indígenas de la ONU, de 2007, en su Art. 31, reconoce el derecho de los pueblos indígenas a controlar y proteger su patrimonio cultural y sus conocimientos tradicionales, incluyendo los recursos genéticos, así como a proteger y desarrollar su propiedad intelectual sobre dicho patrimonio.

El sentido de dicha regla es el reconocimiento de que los pueblos indígenas tienen derechos de propiedad sobre los conocimientos tradicionales y expresiones culturales generados y creados por ellos, y que los derechos de propiedad indígena sobre las tierras y los recursos naturales usados tradicionalmente se extiende a los recursos genéticos que han usado o poseído tradicionalmente (29:2).

La demanda persistente de los representantes indígenas en las negociaciones de la OMPI en el Comité Intergubernamental sobre Propiedad Intelectual y Recursos Genéticos, Conocimientos Tradicionales y Folclore fueron el impulso, mediante un instrumento o régimen internacional vinculante, al desarrollo de sistemas sui generis que reconozcan el derecho consuetudinario, el consentimiento fundamentado previo de los poseedores del conocimiento, y su carácter de custodios y propietarios de sus propios conocimientos tradicionales(30:221).
3. El rechazo al dominio público sobre los recursos genéticos

Una consecuencia del reclamo de propiedad cultural sobre los recursos genéticos asociados a los conocimientos tradicionales es el rechazo a la propuesta de declarar a dichos recursos como parte del dominio público. Para los indígenas, ellos no demandan nuevos derechos de propiedad sobre recursos que estarían en el dominio público, sino el reconocimiento de derechos preexistentes que han existido dentro de las comunidades.

Desde la perspectiva de los pueblos indígenas, la noción de dominio público opera para negar la legitimidad a sus propias normas sobre el control del conocimiento(31:324). Los argumentos en pro del dominio público del conocimiento indígena nuevamente reducen la capacidad de control y toma de decisiones de los pueblos indígenas sobre su conocimiento. El dominio público funcionaría como categoría normativa que excluye el control de determinados recursos de conocimiento, pero en el caso de los conocimientos tradicionales tendría efectos asimétricos, porque deniega el control de estos conocimientos a aquellos que los crearon, las comunidades indígenas, para permitir que los exploten los grupos que actualmente dominan sobre ellos.

El concepto de "dominio público" funcionaría como parte integrante de las estructuras normativas dominantes, para justificar inadvertidamente la denegación de las demandas legítimas de propiedad cultural de los pueblos indígenas para controlar los intangibles(31:325). Por ello, la declaración de que ese conocimiento como dominio público equivaldría a una expropiación de conocimiento.

Del mismo modo que los territorios indígenas fueron declarados Terra Nullius en el proceso de colonización, permitiendo su adquisición por el Estado y los colonizadores, el sistema de derechos de propiedad intelectual ha tratado los conocimientos tradicionales como Gnaritas Nullius conocimiento de nadie-, implicando que caigan en el dominio público(32:33).

El argumento de la Terra Nullius sirvió para denegar los derechos previos de los habitantes origina- 
les de los mundos que fueron conquistados y colonizados por los europeos(8). Antes que titulares de derechos, los indígenas fueron tratados como niños o animales, y los territorios que habitaban pudieron ser ocupados por los europeos como tierras vacías de personas, vacantes y sin uso, de manera que sus invasiones fueron descritas como descubrimientos, la usurpación como comercio y el exterminio y esclavización como misión civilizadora(8). Así también, la propuesta del dominio público sobre los recursos genéticos serviría para denegar la autonomía de los procesos bioculturales y someterlos así a control de las élites estatales.

El carácter discriminatorio del argumento $T_{e}-$ rra Nullius es evidente, como queda refrendado en casos judiciales como el caso George Rodney Burt(34), en el cual un norteamericano obtuvo reconocimiento de la propiedad que le habían vendido los nativos de Islas Fiji antes de su anexión por el Imperio Británico, mientras que esos nativos vieron denegada su propiedad, considerándose sus tierras como Terra Nullius.

Precisamente, el estado actual del Derecho internacional, mediante la afirmación de igualdad, prohibió las prácticas que discriminan por motivos raciales, culturales y étnicos, entre otras razones, de manera que la argumentación de la Terra Nullius, no puede tener cabida en este contexto(35:134). Aquello trae por consecuencia que el Estado no puede emplear concepciones e instituciones únicamente derivadas de las culturas mayoritarias, sino reconocer las formas de propiedad que se han usado históricamente por una cultura particular, considerando sus formas específicas de derechos de propiedad(35:137).

La idea de Terra Nullius ahora es ampliamente entendida, incluso en la cultura legal occidental que lo invocó, no solo como una imposición altamente injusta de los valores de una cultura sobre los de otra, sino como una violación cínica del mismo marco ético occidental(36:164). La denegación del trabajo de las culturas indígenas en el cuidado de las tierras que ocupaban y sus recursos se apoyó en la ausencia de convenciones jurídicas similares a las europeas para asignar derechos de propiedad, de manera que éste no existiría(36). Lo que sucede es que aquella idea constituía, antes que una simple negación de la realidad, una verdadera ficción legal que los juristas del Derecho internacional aplicaban para señalar que la soberanía y el dominio solo podían ser ejercidos o amparados por Estados de pueblos "civilizados" (37:43ss).

Si bien la idea de Terra Nullius estuvo más claramente a la base de la conquista anglosajona que de la ibérica, en la que se fueron configurando instituciones fronterizas y se reconocía de manera asimétrica la realidad de los pueblos indígenas, una vez configurada la conquista o, a más tardar, cuando se instalan las repúblicas poscoloniales, se producen consecuencias similares cuando se deniegan las instituciones y los sistemas normativos indígenas(38). El colonialismo interno de las elites de los nuevos Estados sucesores del gobierno colonial concibió las tierras internas sobre las que pretendía soberanía como tierras salvajes, no ocupadas de manera civilizada, y fueron tomadas entonces por las empresas de construcción del Estado en América Latina, de una manera comparable a las colonizaciones anglosajonas(39:150ss).

El carácter colectivo y a menudo anónimo de los conocimientos tradicionales, así como la naturaleza incremental o gradual de su evolución, hace que no sea concebible, en la lógica de los derechos de propiedad intelectual, atribuir la propiedad de tales conocimientos a un individuo o a un grupo identificable de individuos dentro de los pueblos indígenas, de manera que no es considerada una obra protegida y cae, en aquella misma lógica, dentro del dominio público(35:147;40:64-66).

Por otro lado, la propuesta del dominio público implica que conocimientos tradicionales, que tienen un sentido sagrado o de acceso restringido a no miembros o no iniciados en una práctica religiosas, serán abiertos para el acceso y disposición de cualquiera, en contra de lo prescribe el derecho consuetudinario de los pueblos indígenas para tales clases de conocimientos tradicionales (41:2083ss).

La solución del dominio público sobre los recursos genéticos contradeciría el deseo de los pueblos indígenas de mantener la identidad cultural y espiritual, disociando éstas de sus sistemas de conocimiento y con los bienes que producen. 
4. Críticas a la propiedad cultural y defensa de solución de dominio público

Las críticas de los pueblos indígenas a la solución del dominio público contrastan con la reivindicación de este concepto del régimen de propiedad intelectual para proteger los conocimientos tradicionales.

La consecuencia principal de los reclamos de los pueblos indígenas ha sido la construcción de regímenes de propiedad intelectual sui generis, que atribuyen derechos a los pueblos y comunidades indígenas (vg. Perú, Panamá, Costa Rica, Tailandia). Mientras, los partidarios de considerar a la información genética como un recurso común rechazan los cercamientos del conocimiento que supone constituir derechos de propiedad(42), aunque se refieran a propiedad cultural indígena.

Desde luego, es problemático para la teoría legal que se pueda adquirir el acceso al conocimiento tradicional y éste permanezca en propiedad del titular original(13:249), permitiéndose a lo más un licenciamiento.

Las objeciones que se presentan ante los derechos colectivos en su conjunto pueden hacer valer contra una propiedad cultural sobre los recursos genéticos, en cuanto otorga poderes a una comunidad que pueden ser ejercidos por una mayoría interna, en desmedro de grupos marginalizados dentro de las comunidades y pueblos indígenas(43).

Por otro lado, Michael Brown ha conducido una crítica sistemática a las nociones de propiedad cultural y similares que otorgan control a los pueblos indígenas sobre los conocimientos tradicionales, en cuanto reivindican una lógica normativa occidental de la propiedad y ofrecen soluciones estandarizadas y uniformes, tal y como el sistema de derechos de propiedad intelectual que cuestionan(43:59). Adicionalmente, los derechos de propiedad favorecerían un control de la cultura en sentido preservacionista, que oscurecería su dinamismo, apropiación, hibridaciones y contaminaciones(44).

Frente a ello, Rosemary Coombe indica que las objeciones antropológicas a los usos de conceptos occidentales, como en el caso de la propiedad, re- ducen con mucha facilidad a los pueblos indígenas como un agente inducido, perdiendo de vista las oportunidades de acción colectiva que se les abren y la forma que tienen de ocuparlas(45:114).

Okejidi (46), por su parte, reivindica las posibilidades del dominio público, atendiendo a su naturaleza plural y su carácter abierto para ser determinado por la legislación doméstica, permitiendo pensar en un dominio público hecho a la medida para para proteger los conocimientos tradicionales, a la vez que despeja las objeciones de científicos e inversionistas a la propiedad intelectual de los pueblos indígenas como un control que funcionaría como obstáculo a la innovación. Aquel dominio público podría establecer obligaciones en el uso de los conocimientos que distinga entre sus diversos tipos, según su grado de accesibilidad: conocimiento tradicional sagrado o mantenido en secreto en que debe otorgarse derechos económicos y morales; conocimiento tradicional estrechamente sostenido, que también debería otorgar derechos económicos y morales; conocimiento tradicional ampliamente difundido, que debería implicar atribución, y conocimiento tradicional genérico, que no implicaría ningún derecho.

Las dilatadas negociaciones dentro del IGC, después de 18 años, ofrecen pocas perspectivas favorables a las pretensiones de los pueblos indígenas, inclinándose hasta ahora por desarrollar una protección defensiva de los conocimientos tradicionales, dejando de lado la protección positiva mediante derechos de propiedad sui generis sobre dichos conocimientos. Con dicho fin, el Art 5 bis. 4a de la 2a versión revisada (2016) del proyecto de convención establece que las partes deberán "[facilitar/fomentar], cuando proceda, la creación, intercambio y difusión de bases de datos [accesibles al público] de recursos genéticos y conocimientos tradicionales asociados a los recursos genéticos, y el acceso a dichas bases de datos" (47).

En 2018, EE.UU., Canadá, Noruega, Japón y Corea del Sur promovieron una "Recomendación Conjunta sobre los Recursos Genéticos y los Conocimientos Tradicionales Asociados". En dicho documento se proponen como objetivo prevenir las patentes erróneamente garantizadas para invenciones que no sean nuevas ni impliquen acti- 
vidad inventiva respecto de los recursos genéticos y los conocimientos tradicionales asociados a los recursos genéticos(48).

\section{Conclusión}

Los pueblos indígenas han sostenido consistentemente una posición en los foros internacionales, especialmente en los debates de las reuniones del Convenio sobre la Diversidad Biológica y en las reuniones del Comité Intergubernamental de la OMPI, en los que han defendido su propiedad cultural sobre los recursos genéticos asociados a los conocimientos tradicionales. Dicha propiedad se caracteriza por su fundamento en la persistencia de su identidad y por ser permanente, colectiva y estructurada según su derecho consuetudinario, diferenciándose de las nociones occidentales de propiedad.
Con la reivindicación de la propiedad intelectual, los pueblos indígenas descartan la solución del dominio público como riesgosa para la continuidad de sus formas de vida.

Estas demandas han encontrado acogida en el régimen de derechos humanos, que se expresa en la Declaración de la ONU de derechos de los pueblos indígenas.

Sin embargo, esta posición tiene cuestionamientos y parece no lograr abrirse paso en las negociaciones del Comité de la OMPI. Si se concretara allí un acuerdo internacional en los términos vigentes, generará inevitablemente una futura colisión de regímenes internacionales entre la regulación de la propiedad intelectual y los derechos humanos de los pueblos indígenas. 
Recursos genéticos y pueblos indígenas - Salvador Millaleo Hernández

\section{Referencias}

1. Lawson Ch, Adhikari K. Biodiversity, Genetic Resources and Intellectual Property. In: Lawson Ch, Adhikari K, (eds.). Biodiversity, Genetic Resources and Intellectual Property, Developments in Access and Benefit Sharing. New York: Routledge; 2018: 1-8.

2. Herrera S, Rodríguez E. Etnoconocimiento en Latinoamérica. Apropiación de Recursos Genéticos y Bioética. Acta Bioethica 2004; 10(2): 181-190.

3. Srinivas K. Intellectual property rights and bio commons: open source and beyond. International Social Science Journal 2006; 58(188): 319-334.

4. Raustiala K, Victor D. The Regime Complex for Plant Genetic Resources. International Organization 2004; 58(2): $277-309$.

5. Safrin S. Hyperownership in a Time of Biotechnological Promise: The International Conflict to Control the Building Blocks of Life. American Journal of International Law 2004; 98(4): 641-685.

6. Rhodes C. Potential International Approaches to Ownership/Control of Human Genetic Resources. Health Care Analysis 2016; 24(3): 260-277.

7. Correa C. Sovereign and Property Rights over Plant Genetic Resources. Commission on Plant Genetic Resources, First Extraordinary Session, Rome, 7-11 November 1994. Roma: FAO; 1994.

8. Shiva V. The Seed and the Earth, Biotechnology and the Colonization of Regeneration. Canadian Woman Studies 1993; 13(3): 23-27.

9. Shiva V, Jafri A, Gitanjali B, Holla-Bhar R, Rhadha R. The Enclosure and Recovery of the Commons: Biodiversity, Indigenous Knowledge and Intellectual Property Rights. New Delhi: Research Foundation for Science, Technology and Ecology; 1997.

10. Stoll T. Intellectual Property and Technologies. In: Hohmann J, Weller M, (eds.). The UN Declaration on the Rights of Indigenous Peoples, A Commentary. Oxford: Oxford University Press; 2018: 299-327.

11. Tsosie, R. Indigenous Peoples' Claims to Cultural Property: A Legal Perspective. Museum Anthropology 1997; $21:$ 5-11.

12. Tsosie R. International trade in indigenous cultural heritage: An argument for indigenous governance of cultural property. In: Beat Ch, Kuprecht K, Lai J, (eds.). International Trade in Indigenous Cultural Heritage: Legal and Policy Issues. Cheltenham: Edward Elgar Publishing; 2012: 221-245.

13. Aguilar G. Access to genetic resources and protection of traditional knowledge in the territories of indigenous peoples. Environmental Science \& Policy 2001; 4(4-5): 241-256.

14. IGC. Report of the second session. WIPO Intergovernmental Committee on Intellectual Property and Genetic Resources, Traditional Knowledge and Folklore, December 14, 2002, WIPO/GRTKF/IC/2/16. Ginebra: WIPO; 2002.

15. Berman T. "As Long as the Grass grows": Representing indigenous claims. In: Riley M, (ed.). Indigenous Intellectual Property Rights, Legal Obstacles and Innovative Solutions. Walnut Creek et al.: Altamira Press; 2004: 3-25.

16. Kuprecht K. Indigenous Peoples' Cultural Property Claims. Repatriation and Beyond. Springer; 2014.

17. Carpenter K, Katyal S, Riley A. In Defense of Property. The Yale Law Journal 2009; 118(6): 1022-1125.

18. Posey D. Mind the Gaps: Identifying Commonalities and Divergencies between Indigenous Peoples and Farmers Groups. Conference Protecting Knowledge: Traditional Resource Rights in the New Millennium. February 23rd - February 26th, 2000. Vancouver: The Union of British Columbia Indian Chiefs; 2000.

19. Tobin B. Indigenous Peoples, Customary Law and Human Rights - Why Living Law Matters. New York: Earthscan; 2014.

20. Nijar G. In defense of local community knowledge and biodiversity: A conceptual framework and the essential elements of a rights regime. Penang: Third World Network; 1996.

21. Halewood M. Common Law Aboriginal Knowledge Protection Rights: Recognizing the Rights of Aboriginal Peoples in Canada to Prohibit the Use and Dissemination of Elements of their Knowledge. Doctoral Thesis. Toronto: York University; 2005.

22. Bondía D. La reivindicación de los derechos humanos emergentes bioculturales: los logros del protocolo de Nagoya. In: Pons X. (ed.). Alimentación y Derecho Internacional, Normas, Instituciones y Procesos. Madrid: Marcial Pons; 2013: 229-252.

23. Oguamanam Ch. Intellectual Property Rights in Plant Genetic Resources: Farmers' Rights and Food Security of Indigenous and Local Communities. Drake Journal of Agricultural Law 2006; 11(3): 273- 305.

24. 24. Carta de la Tierra de los Pueblos Indígenas; 1992. Disponible en: http://ayi-noticias.blogspot.com/p/declaracion-kari-ocay-la-carta-de-la.html [Acceso 16 de septiembre de 2018]

25. 25. Declaración de Mataatua de los derechos intelectuales y culturales de los pueblos indígenas; 1993. Disponible en: http:// indigenas.bioetica.org/leyes/doc23.htm [Acceso 16 de septiembre de 2018]

26. 26. Declaración de Kimberley de la Cumbre Internacional de los Pueblos Indígenas sobre Desarrollo Sostenible en el Territorio KhoiSan; 2002. Disponible en: http://www.nacionmulticultural.unam.mx/movimientosindigenas/docs/82.pdf [Acceso 16 de septiembre de 2018] 
27. Octava Conferencia de las Partes del Convenio sobre la Diversidad Biológica, Plenaria del 31 de marzo de 2006. Disponible en: https://www.servindi.org/actualidad/467 [Acceso 16 de septiembre de 2018]

28. 28. Declaración de la Alianza de los Guardianes e Hijos de la Madre Tierra de Brasilia; 2017. Disponible en: http://allianceofguardians.org/doc/call2017/AGMN_Apelo-Internacional-2017-\&-Anexo_ES.pdf [Acceso 16 de septiembre de 2018]

29. Anaya J. Examen técnico de algunas cuestiones esenciales de propiedad intelectual de los proyectos de instrumentos de la OMPI relativos a los recursos genéticos, los conocimientos tradicionales y las expresiones culturales tradicionales. WIPO/GRTKF/IC/33/INF/9. Ginebra: WIPO; 2016.

30. Solomon M. An indigenous perspective on the WIPO IGC. In: Robinson D, Abdel-Latif A, Roffe P, (eds.). Protecting Traditional Knowledge, The WIPO Intergovernmental Committee on Intellectual Property and Genetic Resources, Traditional Knowledge and Folklore. New York: Routledge; 2017: 219-229.

31. Van Caenegen W. The Public Domain: Scientia Nullius? European Intellectual Property Review 2002; 24(6): 324-330.

32. Bratspies R. The New Discovery Doctrine: Some Thoughts on Property Rights and Traditional Knowledge. American Indian Law Review 2007; 31(2): 315-340.

33. Younging G. Gnaritas Nullius (No One's Knowledge): The Essence of Traditional Knowledge and Its Colonization through Western Legal Regimes. In: Elliot P, Hepting D. (eds.). Free Knowledge, Confronting the Commodification of Human Discovery. Regina: University of Regina Press; 2015: 149-179.

34. Case George Rodney Burt (United States) v. Great Britain (Fijian Land Claims), British American Claims Tribunal, 26 October 1923. In: UNO, Reports of International Arbitral Awards, Vol. VI. New York: UNO; 2006: 93-99.

35. Åhrén, M. Indigenous Creativity and the Public Domain - Terra Nullius Revisited? In: Xanthaki A, Valkonen S, Heinämäki L, Nuorgam P. (eds.). Indigenous Peoples' Cultural Heritage Rights, Debates, Challenges. Leiden: Brill Nijhoff; 2017: 130-148.

36. Brunk C. Appropriation of Traditional Knowledge: Ethics in the Context of Ethnobiology, Part II: Philosophical and Ethical Issues: Toward the Creation of 'Ethical Space'. In: Young J, Brunk C. (eds.). The Ethics of Cultural Appropriation. Malden MA \& Oxford: Wiley-Blackwell; 2009: 161-172.

37. Gilbert J. Indigenous Peoples' Land Rights under International Law, From Victims to Actors. Leiden: Brill Nijhoff; 2016.

38. Calbucura J. La descolonización del saber y el ser mapuche: un caso de estudio al celebrarse el bicentenario de la construcción de la República de Chile. Polis, Revista Latinoamericana 2013; 12(35): 405-427.

39. Hendlin Y. From Terra Nullius to Terra Communis: Reconsidering Wild Land in an Era of Conservation and Indigenous Rights. Environmental Philosophy 2014; 11(2): 141-174.

40. Carpenter M. Intellectual Property Law and Indigenous Peoples: Adapting Copyright Law to the Needs of a Global Community. Yale Human Rights Law \& Development Law Journal 2004; 7(1): 51-78.

41. Younging G. Traditional Knowledge Exists; Intellectual Property is Invented or Created. University of Pennsylvania Journal of International Law, 2015; 36(4): 1077-1085.

42. Lucchi N. Understanding genetic information as a common: From bioprospecting to personalized medicine. International Journal of the Commons 2013; 7(2): 313-338.

43. Brown M. Heritage as Property. In: Verdery K, Humphrey C. (eds.). Property in Question: Value Transformation in the Global Economy. Oxford: Berg Publishers; 2004: 49-68.

44. Mezey N. The Paradoxes of Cultural Property. Columbia Law Review 2007; 107(8): 2004-2046.

45. Coombe R. 'Possessing Culture': Political Economies of Community Subjects and their Properties. In: Strang S, Busse M. (eds.). Ownership and Appropriation. Oxford: Berg Publishers; 2011: 105-127.

46. Okediji R. Traditional Knowledge and the Public Domain. CIGI Papers No 176-June 2018. Waterloo, Canada: Centre for International Governance Innovation; 2018.

47. IGC. La Protección de los Conocimientos Tradicionales: Proyecto de Artículos, Rev. 2, 2016. WIPO/GRTKF/IC/37/4. Ginebra: WIPO; 2018.

48. IGC. Recomendación Conjunta sobre los Recursos Genéticos y los Conocimientos Tradicionales Asociados, 6 de agosto de 2018, WIPO/GRTKF/IC/37/12. Ginebra: WIPO; 2018.

Recibido: 17 de octubre de 2018

Aceptado: 3 de diciembre de 2018 